\title{
Green function for hyperbolic media
}

\author{
Andrey S. Potemkin, ${ }^{1}$ Alexander N. Poddubny, ${ }^{1,2}$ Pavel A. Belov,${ }^{1,3}$ and Yuri S. Kivshar ${ }^{1,4}$ \\ ${ }^{1}$ Department of Photonics and Optoinformatics, National University of Information Technology, Mechanics and Optics (ITMO), \\ St. Petersburg 197101, Russia \\ ${ }^{2}$ Ioffe Physical-Technical Institute of the Russian Academy of Sciences, St. Petersburg 194021, Russia \\ ${ }^{3}$ School of Electronic Engineering and Computer Science, Queen Mary University of London, Mile End Road, \\ London E1 4NS, United Kingdom \\ ${ }^{4}$ Nonlinear Physics Center, Research School of Physics and Engineering, Australian National University, Canberra ACT 0200, Australia
}

(Received 29 June 2012; published 28 August 2012)

\begin{abstract}
We revisit the problem of the electromagnetic Green function for homogeneous hyperbolic media, where longitudinal and transverse components of the dielectric permittivity tensor have different signs. We analyze the dipole emission patterns for both dipole orientations with respect to the symmetry axis and for different signs of dielectric constants, and show that the emission pattern is highly anisotropic and has a characteristic crosslike shape: the waves are propagating within a certain cone and are evanescent outside this cone. We demonstrate the coexistence of the conelike pattern due to emission of the extraordinary TM-polarized waves and elliptical pattern due to emission of ordinary TE-polarized waves. We find a singular complex term in the Green function, proportional to the $\delta$ function and governing the photonic density of states and Purcell effect in hyperbolic media.
\end{abstract}

DOI: 10.1103/PhysRevA.86.023848

PACS number(s): 42.50.-p, 74.25.Gz, 78.70.-g

\section{INTRODUCTION}

Hyperbolic medium is a particular case of uniaxial anisotropic dielectric medium where the main values of the permittivity tensor $\hat{\varepsilon}$ have opposite signs [1], and the isofrequency surface of extraordinary waves is a hyperboloid. The recent attention of researchers to the hyperbolic metamaterials stems from their unique optical properties allowing negative refraction, hyperlensing, and cloaking phenomena [2-4]. They are also very promising for quantum nanophotonics [5,6], because the density of states, determined by the hyperboloid area, is divergent. This means an infinite spontaneous decay rate of a quantum emitter embedded in a hyperbolic medium, i.e., infinite Purcell effect [7]. In the realistic case, the radiation rate is limited by certain cutoffs in the wave-vector space [8-12], however, experimental observation of radiative enhancement is still possible $[5,13]$.

A general hyperbolic medium can be characterized by a dielectric permittivity tensor

$$
\hat{\varepsilon}=\left(\begin{array}{ccc}
\varepsilon_{\perp} & 0 & 0 \\
0 & \varepsilon_{\perp} & 0 \\
0 & 0 & \varepsilon_{\|}
\end{array}\right), \quad \operatorname{Re} \varepsilon_{\perp} \operatorname{Re} \varepsilon_{\|}<0 .
$$

The resulting isofrequency surface of extraordinary waves is a hyperboloid,

$$
\frac{q_{x}^{2}+q_{y}^{2}}{\varepsilon_{\|}}+\frac{q_{z}^{2}}{\varepsilon_{\perp}}=\left(\frac{\omega}{c}\right)^{2}
$$

Two different types of hyperboloids are possible for $\varepsilon_{\|}<0$, $\varepsilon_{\perp}>0$ [see Fig. 1(a)] and for $\varepsilon_{\|}>0, \varepsilon_{\perp}<0$ [see Fig. 1(b)].

The hyperbolic medium can be realized in several ways. The first realization has been reported for magnetized plasma in the microwave spectral range [14]. Under a very strong static magnetic field applied along the $z$ axis the plasma is described by the permittivity with $[14,15]$

$$
\varepsilon_{\perp}=1, \quad \varepsilon_{\|}=1-\frac{\omega_{p}^{2}}{\omega^{2}} .
$$

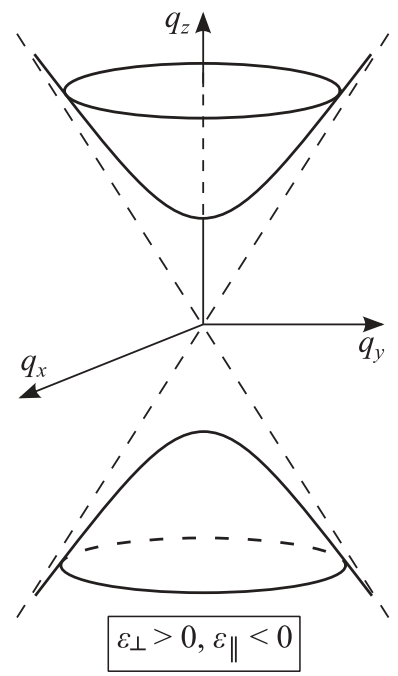

(a)

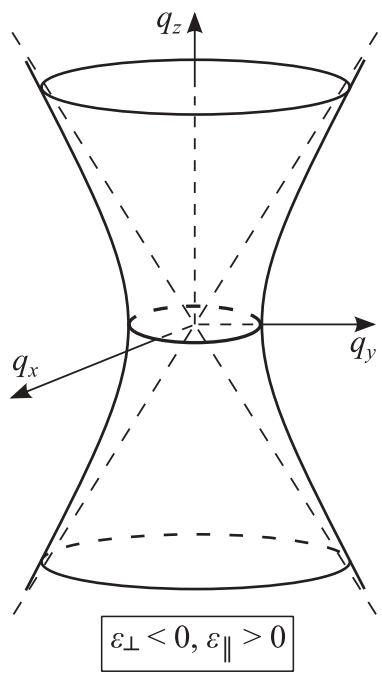

(b)
FIG. 1. Schematic illustration of the isofrequency surface in the wave-vectors space for the hyperbolic medium with $\varepsilon_{\|}<0, \varepsilon_{\perp}>0$ (a) and with $\varepsilon_{\|}<0, \varepsilon_{\perp}>0$ (b).

Thus, the frequencies $\omega$ below the plasma frequency $\omega_{p}$ correspond to the hyperbolic regime with $\varepsilon_{\|}<0$.

The interest in the hyperbolic medium is now revived and rapidly increases due to its successful realization using artificial photonic structures, metamaterials [5,16-19]. In particular, the layered structure composed of alternating dielectric and metallic slabs is described by effective permittivities

$$
\varepsilon_{\perp}=\frac{\varepsilon_{1} d_{1}+\varepsilon_{2} d_{2}}{d_{1}+d_{2}}, \quad \varepsilon_{\|}=\left(\frac{\varepsilon_{1}^{-1} d_{1}+\varepsilon_{2}^{-1} d_{2}}{d_{1}+d_{2}}\right)^{-1},
$$

where $\varepsilon_{1}, \varepsilon_{2}, d_{1}, d_{2}$ are dielectric constants and thicknesses of slabs, respectively. Since the dielectric constants of the metals in the optical frequency range are negative, it is possible to adjust the thicknesses of slabs in order to obtain either $\varepsilon_{\perp}<0$ 
or $\varepsilon_{\|}<0$ [20,21]. Realizations of the hyperbolic regime have been also reported for metamaterials based on nanorod arrays [17,22,23] and for graphite [24].

The ongoing studies of the hyperbolic medium raise the demand for the general theoretical formalism. The most rigorous description of the optical properties of the arbitrary photonic structure is provided by its tensor Green function [25], determined from

$$
\left(\nabla \times \nabla \times \hat{I}-k^{2} \hat{\varepsilon}\right) \hat{G}(\mathbf{r})=4 \pi k^{2} \hat{I} \delta(\mathbf{r}),
$$

where $\hat{I}$ is the unit tensor and $k=\omega / c$. The Green function for the medium with uniaxial permittivity tensor is presented in a number of works [1,26-34]. The most general form of the result is given by Chen in Ref. [27] in dyadic form and by Savchenko in Ref. [33] in Cartesian form. However, despite the impressive amount of the research done, we are not aware of any comprehensive study of the Green function in the hyperbolic case. Moreover, all existing works except [30,32] neglect the singular term in the Green function, which, as will be shown below, is crucial for the description of the photonic density of states and Purcell enhancement in the hyperbolic regime. Here we present a general theory of the Green function and dipole emission in the hyperbolic medium. We analyze both types of hyperbolic medium, illustrated in Fig. 1, and both axial and transverse dipole orientations.

The rest of the paper is organized as follows. Section II outlines the Green function calculation. The singular term in the Green function is discussed in Sec. III. Section IV presents the calculated emission patterns of point dipole. The main paper results are summarized in Sec. V. Auxiliary expressions are presented in the Appendixes.

\section{GREEN-FUNCTION CALCULATION}

The Green function (3) may be calculated either in real space via the methods of operators [31] or in wave-vector space via Fourier analysis [27]. The Fourier representation of $\hat{G}(\mathbf{r})$, defined from

$$
\hat{G}(\mathbf{r})=\int \frac{d^{3} q}{(2 \pi)^{3}} e^{i \mathbf{q} \cdot \mathbf{r}} \hat{G}(\mathbf{q}),
$$

is given by

$$
\begin{aligned}
\hat{G}(\mathbf{q})= & 4 \pi k^{2}\left\{\left(\varepsilon_{\perp} \varepsilon_{\|} \hat{\varepsilon}-\frac{\mathbf{q} \otimes \mathbf{q}}{k^{2}}\right) \frac{1}{q_{\|}^{2} \varepsilon_{\|}+q_{\perp}^{2} \varepsilon_{\perp}-k^{2} \varepsilon_{\perp} \varepsilon_{\|}}\right. \\
& +\frac{(\mathbf{q} \times \hat{\mathbf{z}}) \otimes(\mathbf{q} \times \hat{\mathbf{z}})}{q_{\perp}^{2}}\left(\frac{1}{q^{2}-k^{2} \varepsilon_{\perp}}\right. \\
& \left.\left.-\frac{\varepsilon_{\|}}{q_{\|}^{2} \varepsilon_{\|}+q_{\perp}^{2} \varepsilon_{\perp}-k^{2} \varepsilon_{\perp} \varepsilon_{\|}}\right)\right\}
\end{aligned}
$$

Here $q_{\|} \equiv q_{z}$ and $q_{\perp}^{2}=q_{x}^{2}+q_{y}^{2}$. The symbol $\otimes$ denotes the direct product, $[\mathbf{a} \otimes \mathbf{b}]_{\alpha \beta} \equiv a_{\alpha} b_{\beta}$. The poles in Eq. (5) determine the dispersion equations of the electromagnetic waves. Two poles correspond to extraordinary (TM) waves, with magnetic field $\mathbf{H}$ perpendicular to $z$ axis, and to ordinary (TE) waves, with $\mathbf{E} \cdot \hat{\mathbf{z}}=\mathbf{0}$ and $q=k \sqrt{\varepsilon_{\perp}}$.
Both direct and reciprocal-space methods give the same following result for the Green function [27]:

$$
\begin{aligned}
\hat{G}(\mathbf{r})= & \frac{1}{\sqrt{\varepsilon_{\perp}}}\left\{\left(k^{2} \hat{\epsilon}+\nabla \otimes \nabla\right) \frac{e^{i k r_{e}}}{r_{e}}\right. \\
& +k^{2}\left(\varepsilon_{\perp} \frac{e^{i k r_{o}}}{r_{o}}-\varepsilon_{\|} \frac{e^{i k r_{e}}}{r_{e}}\right) \frac{(\mathbf{r} \times \hat{\mathbf{z}}) \otimes(\mathbf{r} \times \hat{\mathbf{z}})}{(\mathbf{r} \times \hat{\mathbf{z}})^{2}} \\
& \left.-i k \frac{e^{i k r_{o}}-e^{i k r_{e}}}{(\mathbf{r} \times \hat{\mathbf{z}})^{2}}\left(\hat{\mathbf{I}}-\hat{\mathbf{z}} \otimes \hat{\mathbf{z}}-\frac{2(\mathbf{r} \times \hat{\mathbf{z}}) \otimes(\mathbf{r} \times \hat{\mathbf{z}})}{(\mathbf{r} \times \hat{\mathbf{z}})^{2}}\right)\right\},
\end{aligned}
$$

where

$$
\begin{aligned}
& \hat{\epsilon} \equiv \varepsilon_{\perp} \varepsilon_{\|} \hat{\varepsilon}^{-1}=\varepsilon_{\|}(\hat{\mathbf{I}}-\hat{\mathbf{z}} \otimes \hat{\mathbf{z}})+\varepsilon_{\perp} \hat{\mathbf{z}} \otimes \hat{\mathbf{z}}, \\
& r_{e}=\sqrt{\mathbf{r}(\hat{\epsilon} \mathbf{r})}=\sqrt{\varepsilon_{\|}\left(x^{2}+y^{2}\right)+\varepsilon_{\perp} z^{2}}, \quad r_{o}=\sqrt{\varepsilon_{\perp}} r .
\end{aligned}
$$

This result was obtained without any assumptions for signs of real of parts $\varepsilon_{\perp}$ and $\varepsilon_{\|}$and is applicable to case of the hyperbolic medium. Imaginary parts of $\varepsilon_{\perp}$ and $\varepsilon_{\|}$and of the square roots in expressions for $r_{e}$ and $r_{o}$ should be positive (we assume the time dependence $e^{-i \omega t}$ ).

Equation (6) is not the final result for the Green function yet, because the derivative in the term $\nabla \otimes \nabla e^{i k r_{e}} / r_{e}$ is not evaluated. Some authors [31,34] leave the expression for $\hat{G}(\mathbf{r})$ in the form (6) without taking this derivative. However, this term produces singularity in $\hat{G}(\mathbf{r})$. The nature of this singularity is the same for the well-known identity

$$
\Delta \frac{1}{r}=-4 \pi \delta(\mathbf{r})
$$

Calculating the derivative we obtain the final expression of singular Green function:

$$
\begin{aligned}
\hat{G}(\mathbf{r})= & \hat{G}_{\text {sing }}(\mathbf{r})+\frac{1}{\sqrt{\varepsilon_{\perp}}}\left\{k^{2} \frac{e^{i k r_{e}}}{r_{e}}\left(1-\frac{1}{i k r_{e}}-\frac{1}{k^{2} r_{e}^{2}}\right) \hat{\epsilon}\right. \\
& -k^{2} \frac{e^{i k r_{e}}}{r_{e}^{3}}\left(1-\frac{3}{i k r_{e}}-\frac{3}{k^{2} r_{e}^{2}}\right)(\hat{\epsilon} \mathbf{r}) \otimes(\hat{\epsilon} \mathbf{r}) \\
& +k^{2}\left(\varepsilon_{\perp} \frac{e^{i k r_{o}}}{r_{o}}-\varepsilon_{\|} \frac{e^{i k r_{e}}}{r_{e}}\right) \frac{(\mathbf{r} \times \hat{\mathbf{z}}) \otimes(\mathbf{r} \times \hat{\mathbf{z}})}{(\mathbf{r} \times \hat{\mathbf{z}})^{2}} \\
& \left.-i k \frac{e^{i k r_{o}}-e^{i k r_{e}}}{(\mathbf{r} \times \hat{\mathbf{z}})^{2}}\left(\hat{\mathbf{I}}-\hat{\mathbf{z}} \otimes \hat{\mathbf{z}}-\frac{2(\mathbf{r} \times \hat{\mathbf{z}}) \otimes(\mathbf{r} \times \hat{\mathbf{z}})}{(\mathbf{r} \times \hat{\mathbf{z}})^{2}}\right)\right\},
\end{aligned}
$$

where $\hat{G}_{\text {sing }}(\mathbf{r})$ stands for the singular contribution,

$$
\begin{aligned}
\hat{G}_{\text {sing }}(\mathbf{r}) & =\left[(\nabla \otimes \nabla) \frac{1}{\sqrt{\varepsilon_{\perp}} r_{e}}\right]_{\text {sing }} \\
& \equiv-4 \pi\left[\int \frac{d^{3} q}{(2 \pi)^{3}} e^{i \mathbf{q} \cdot \mathbf{r}} \frac{\mathbf{q} \otimes \mathbf{q}}{q_{\|}^{2} \varepsilon_{\|}+q_{\perp}^{2} \varepsilon_{\perp}}\right]_{\text {sing }} .
\end{aligned}
$$

In the general case we failed to obtain a closed answer for $\hat{G}_{\text {sing }}$ via ordinary functions and the $\delta$ function. Equation (8) should be understood instead only as a generalized function, i.e., only its convolutions with ordinary test functions are relevant [35]. The singular Green function is essential in the hyperbolic case: while in the elliptic regime it leads only to the radiative corrections to the resonance frequency, in the 
hyperbolic regime it provides a diverging contribution to the photonic density of states and the Purcell factor.

The answers above are valid for both hyperbolic and elliptic media with arbitrary signs of real parts of dielectric constants. In the following Secs. III and IV we analyze singular and regular contributions to the Green function, Eqs. (8) and (7), respectively, in more detail, focusing on the hyperbolic regime.

\section{SINGULAR TERM OF THE GREEN FUNCTION}

In order to stress the importance of the singular term in the Green function we will first show how it contributes to the Purcell factor in the hyperbolic regime. Generally, the Purcell factor of the light emitter oriented along the $\hat{\mathbf{n}}$ direction can be found [36] as

$$
f=\frac{3}{2 k^{3}} \operatorname{Im} \hat{\mathbf{n}} \cdot \hat{G}(0) \hat{\mathbf{n}} .
$$

In the elliptic regime the imaginary part of the Green function, determining the local density of states, stays finite in the point $\mathbf{r}=0$. In the hyperbolic regime it diverges, as will be shown below, and leads to the infinite Purcell factor. As has been indicated in our previous work [8], this divergence is smeared out for a finite-size emitter, characterized with spatial distribution $\Phi(\mathbf{r})$, normalized as $\int d^{3} r \Phi(\mathbf{r})=1$. In the case of the semiconductor quantum dot $\Phi(\mathbf{r})$ is proportional to the exciton envelope function [37]. For the distributed source one has instead of Eq. (9)

$$
f=\frac{3}{2 k^{3}} \hat{\mathbf{n}} \cdot \int d^{3} r d^{3} r^{\prime} \Phi(\mathbf{r}) \Phi\left(\mathbf{r}^{\prime}\right) \hat{G}\left(\mathbf{r}-\mathbf{r}^{\prime}\right) \hat{\mathbf{n}} .
$$

In particular, if the envelope is chosen in the Gaussian form,

$$
\Phi(r)=\frac{1}{(\sqrt{2 \pi} a)^{3}} \exp \left(-\frac{r^{2}}{2 a^{2}}\right),
$$

the Purcell factor for the dipole moment oriented along the $z$ direction reads [8]

$$
f=\sqrt{\varepsilon_{\perp}}-\frac{3 \sqrt{\pi \varepsilon_{\perp}}\left(2 \varepsilon_{\perp}+\left|\varepsilon_{\|}\right|\right)}{8 k a\left(\varepsilon_{\perp}+\left|\varepsilon_{\|}\right|\right)^{3 / 2}}+\frac{3 \sqrt{\pi \varepsilon_{\perp}}}{8(k a)^{3}\left(\varepsilon_{\perp}+\left|\varepsilon_{\|}\right|\right)^{3 / 2}},
$$

where we assumed $\varepsilon_{\perp}>0, \varepsilon_{\|}<0, \operatorname{Im} \varepsilon=0$. This expression has been derived for a source size much smaller than the wavelength, $k a \ll 1$, so that the Purcell factor is governed by the third term in Eq. (12), proportional to $1 /(k a)^{3}$. We will now show that it is the singular contribution to the Green function that solely determines this leading third term.

The explicit form of the generalized function (8) in the anisotropic case can be obtained within the space of the test functions, analytical in the point $\mathbf{r}=0$ :

$$
\begin{aligned}
\hat{\tilde{G}}_{\text {sing }}(\mathbf{r})= & \frac{2 \pi \delta(\mathbf{r})}{\varepsilon_{\|}-\varepsilon_{\perp}}[\hat{\mathbf{x}} \otimes \hat{\mathbf{x}}+\hat{\mathbf{y}} \otimes \hat{\mathbf{y}}-2 \hat{\mathbf{z}} \otimes \hat{\mathbf{z}} \\
& +\arctan \left(\sqrt{\varepsilon_{\|} / \varepsilon_{\perp}-1}\right)\left(\frac{2}{\sqrt{\varepsilon_{\|} / \varepsilon_{\perp}-1}} \hat{\mathbf{z}} \otimes \hat{\mathbf{z}}\right. \\
& \left.\left.-\frac{\varepsilon_{\|}}{\varepsilon_{\perp} \sqrt{\varepsilon_{\|} / \varepsilon_{\perp}-1}}(\hat{\mathbf{x}} \otimes \hat{\mathbf{x}}+\hat{\mathbf{y}} \otimes \hat{\mathbf{y}})\right)\right] .
\end{aligned}
$$

The equivalence between Eqs. (13) and (8) within the space of test functions, analytical in the point $r=0$, is directly shown via real-space integration in spherical coordinates. Let us demonstrate it for the $z z$ component:

$$
\begin{aligned}
& \int_{r<R} d^{3} r\left[G_{\text {sing }}\right]_{z z}(\mathbf{r}) \\
& \quad=\int_{r<R} d^{3} r \frac{\partial^{2}}{\partial z^{2}} \frac{1}{\sqrt{\varepsilon_{\perp}} r_{e}}=\oint_{r=R} d \mathbf{S} \cdot \hat{\mathbf{z}} \frac{\partial}{\partial z} \frac{1}{\sqrt{\varepsilon_{\perp}} r_{e}} \\
& \quad=-2 \pi \sqrt{\varepsilon_{\perp}} \int_{0}^{\pi} d \theta \frac{\sin \theta \cos ^{2} \theta}{\left(\varepsilon_{\|} \sin ^{2} \theta+\varepsilon_{\perp} \cos ^{2} \theta\right)^{3 / 2}} .
\end{aligned}
$$

Performing the integral over $\theta$ we obtain an expression, equal to $\int d^{3} r\left[\widetilde{G}_{\text {sing }}\right]_{z z}(\mathbf{r})$, which finalizes the proof. Equation (13) also follows from the wave-vector-space representation in Eq. (8). In the limit $\varepsilon_{\perp}=\varepsilon_{\|}$Eq. (13) reduces to Eq. (19). Obviously, functional (13) is far more complex than (wrong) Eq. (18). Still, it is valid for a narrower set of test functions than Eqs. (8) and (17).

In the particular case of the lossless hyperbolic medium $\left(\varepsilon_{\|} \varepsilon_{\perp}<0, \operatorname{Im} \varepsilon_{\|}, \varepsilon_{\perp} \rightarrow+0\right)$ the singular part of the Green function acquires a nonzero imaginary part,

$$
\begin{aligned}
\operatorname{Im} \hat{\tilde{G}}_{\text {sing }}(\mathbf{r})= & \frac{2 \pi^{2} \sqrt{\left|\varepsilon_{\perp}\right|}}{\left(\left|\varepsilon_{\perp}\right|+\left|\varepsilon_{\|}\right|\right)^{3 / 2}} \delta(\mathbf{r}) \\
& \times\left[\hat{\mathbf{z}} \otimes \hat{\mathbf{z}}+\frac{\left|\varepsilon_{\|}\right|}{\left|\varepsilon_{\perp}\right|}(\hat{\mathbf{x}} \otimes \hat{\mathbf{x}}+\hat{\mathbf{y}} \otimes \hat{\mathbf{y}})\right] .
\end{aligned}
$$

The singulary in Eq. (15) is a direct consequence of the infinite density of TM modes, Eq. (2). The Green function where this singularity is omitted has wrong analytical properties and cannot be used for nanophotonic applications such as the Purcell factor calculation. The leading contribution to the Purcell factor in the hyperbolic regime, the last term in Eq. (12), is directly determined by the singular density-ofstates contribution, Eq. (15). Indeed, substituting Eqs. (11) and (15) into Eq. (10), one immediately obtains the last term in Eq. (12). This term scales as the inverse volume of the source, reflecting the $\delta$ singularity in Eq. (15). In the lossless elliptic case the singular Green function Eq. (13) stays real, $\operatorname{Im} \hat{\tilde{G}}_{\text {sing }}=$ 0 , quenching the Purcell factor. The physical explanation of this result is that the density-of-states singularity disappears because the area of the elliptic isofrequency surface is finite. Mathematically, one can check that Eq. (13) is real even in the case $0<\varepsilon_{\|}<\varepsilon_{\perp}$, when the expression under the square root is negative:

$$
\frac{\arctan \left(\sqrt{\varepsilon_{\|} / \varepsilon_{\perp}-1}\right)}{\sqrt{\varepsilon_{\|} / \varepsilon_{\perp}-1}}=\frac{\operatorname{arctanh} \sqrt{1-\varepsilon_{\|} / \varepsilon_{\perp}}}{\sqrt{1-\varepsilon_{\|} / \varepsilon_{\perp}}},
$$

the right-hand side of Eq. (16) is real because the argument of the inverse hyperbolic tangent function is less than 1.

Equation (13) is equivalent to Eq. (8) only for analytical test functions; in the general case the singular term in the Green function should be treated with special care [38-40]. A closed expression for functional (8) may be explicitly written out only in the isotropic medium with $\varepsilon_{\|}=\varepsilon_{\perp} \equiv \varepsilon$ [41]:

$$
\hat{G}_{\text {sing }}(\mathbf{r})=-\frac{4 \pi}{\varepsilon} \frac{\mathbf{r} \otimes \mathbf{r}}{r^{2}} \delta(\mathbf{r}),
$$

while in the general anisotropic case we have not found a closed-form answer. A previous expression for this functional 
in the anisotropic medium, obtained by Weiglhofer [29,30],

$$
-\frac{4 \pi}{3} \hat{\varepsilon}^{-1} \delta(\mathbf{r})
$$

is obviously wrong since it has been obtained as a naive generalization of the result [42],

$$
-\frac{4 \pi}{3 \varepsilon} \delta(\mathbf{r}),
$$

for the isotropic medium. Despite the fact that functional (19) is frequently used [42], it gives different results than Eq. (17) when applied to functions, nonanalytical in the point $\mathbf{r}=0$, such as $\mathbf{r} \otimes \mathbf{r} / r^{2}$. This discrepancy is discussed in Ref. [41] in detail. Since angular averaging of $\mathbf{r} \otimes \mathbf{r}$ equals $1 / 3$, both functionals (19) and (17) give the same result for a wide range of test functions. However, a straightforward generalization of Eq. (19) to Eq. (18) is not valid since not all rules of normal calculus may be applied to generalized functions [35]. Instead one should use Eq. (13) or the general expression Eq. (8), depending on the test functions.

\section{DIPOLE EMISSION PATTERN}

The Green function (3) allows us to find the electric field for an arbitrary distribution of polarization $\mathbf{P}(\mathbf{r})$,

$$
\mathbf{E}(\mathbf{r})=\int d^{3} r^{\prime} \hat{G}\left(\mathbf{r}-\mathbf{r}^{\prime}\right) \mathbf{P}\left(\mathbf{r}^{\prime}\right) .
$$

For the point dipole $\mathbf{p}$ one has $\mathbf{P}(\mathbf{r})=\mathbf{p} \delta(\mathbf{r})$ and the electric field is given by

$$
\mathbf{E}(\mathbf{r})=\hat{G}(\mathbf{r}) \cdot \mathbf{p} .
$$

In this section we focus on the emission pattern of the point dipole source, which is evaluated at $\mathbf{r} \neq 0$ and hence not affected by the singular $\delta$ term in the Green function discussed above. In the following subsections we consider two principal cases of the orientation of dipole moment $\mathbf{p}$ with respect to the anisotropy axis $\hat{\mathbf{z}}$.

It should be noted that the regular part of the dipole field may be easily obtained without knowledge of the dyadic Green function (7). The electromagnetic field in the uniaxial medium can be decomposed into two parts: the TM field, where $\mathbf{H} \cdot \hat{\mathbf{z}}$ is zero, and the TE field, where $\mathbf{E} \cdot \hat{\mathbf{z}}$ is zero [26]. Solutions for these fields in the hyperbolic medium can be found separately via corresponding solutions in vacuum using the anisotropic scaling method introduced by Clemmow in Ref. [43]. The key point of this method consists in the appropriate scaling of space as well as fields and polarizations from Maxwell's equations in vacuum to obtain expressions for fields and polarizations in the anisotropic medium. The application of Clemmow's method for the hyperbolic medium gives the following scaling rules:

(i) TE polarization

$$
\begin{gathered}
\mathbf{E}(\mathbf{r})=\hat{\epsilon}^{1 / 2} \mathbf{E}_{0}\left(\hat{\epsilon}^{1 / 2} \mathbf{r}\right), \\
\mathbf{H}(\mathbf{r})=\sqrt{\varepsilon_{\perp} \varepsilon_{\|}} \mathbf{H}_{0}\left(\hat{\epsilon}^{1 / 2} \mathbf{r}\right), \\
\mathbf{P}(\mathbf{r})=\sqrt{\varepsilon_{\perp} \varepsilon_{\|}} \hat{\varepsilon}^{1 / 2} \mathbf{P}_{0}\left(\hat{\epsilon}^{1 / 2} \mathbf{r}\right),
\end{gathered}
$$

(ii) TM polarization

$$
\begin{gathered}
\mathbf{E}(\mathbf{r})=\mathbf{E}_{0}\left(\sqrt{\varepsilon_{\perp}} \mathbf{r}\right), \\
\mathbf{H}(\mathbf{r})=\sqrt{\varepsilon_{\perp}} \mathbf{H}_{0}\left(\sqrt{\varepsilon_{\perp}} \mathbf{r}\right), \\
\mathbf{P}(\mathbf{r})=\varepsilon_{\perp} \mathbf{P}_{0}\left(\sqrt{\varepsilon_{\perp}} \mathbf{r}\right),
\end{gathered}
$$

where $\mathbf{E}_{0}, \mathbf{H}_{0}, \mathbf{P}_{0}$ are vacuum solutions and

$$
\begin{aligned}
& \hat{\varepsilon}^{1 / 2}=\sqrt{\varepsilon_{\perp}}(\hat{\mathbf{I}}-\hat{\mathbf{z}} \otimes \hat{\mathbf{z}})+\sqrt{\varepsilon_{\|}} \hat{\mathbf{z}} \otimes \hat{\mathbf{z}}, \\
& \hat{\epsilon}^{1 / 2}=\sqrt{\varepsilon_{\|}}(\hat{\mathbf{I}}-\hat{\mathbf{z}} \otimes \hat{\mathbf{z}})+\sqrt{\varepsilon_{\perp}} \hat{\mathbf{z}} \otimes \hat{\mathbf{z}} .
\end{aligned}
$$

Decomposition of the dipole polarization $\mathbf{P}$ into the $\mathrm{TM} / \mathrm{TE}$ parts is described in $[44,45]$.

\section{A. Dipole parallel to the axis of anisotropy}

Here we consider the case $\mathbf{p}=p \hat{\mathbf{z}}$. Taking into account that

$$
\begin{aligned}
\hat{\epsilon} \cdot \hat{\mathbf{z}} & =\varepsilon_{\perp} \hat{\mathbf{z}}, \quad(\hat{\epsilon} \mathbf{r}) \cdot \hat{\mathbf{z}}=\varepsilon_{\perp} \mathbf{r} \cdot \hat{\mathbf{z}}, \\
(\mathbf{r} \times \hat{\mathbf{z}}) \cdot \hat{\mathbf{z}} & =0, \quad(\hat{\mathbf{I}}-\hat{\mathbf{z}} \otimes \hat{\mathbf{z}}) \cdot \hat{\mathbf{z}}=0,
\end{aligned}
$$
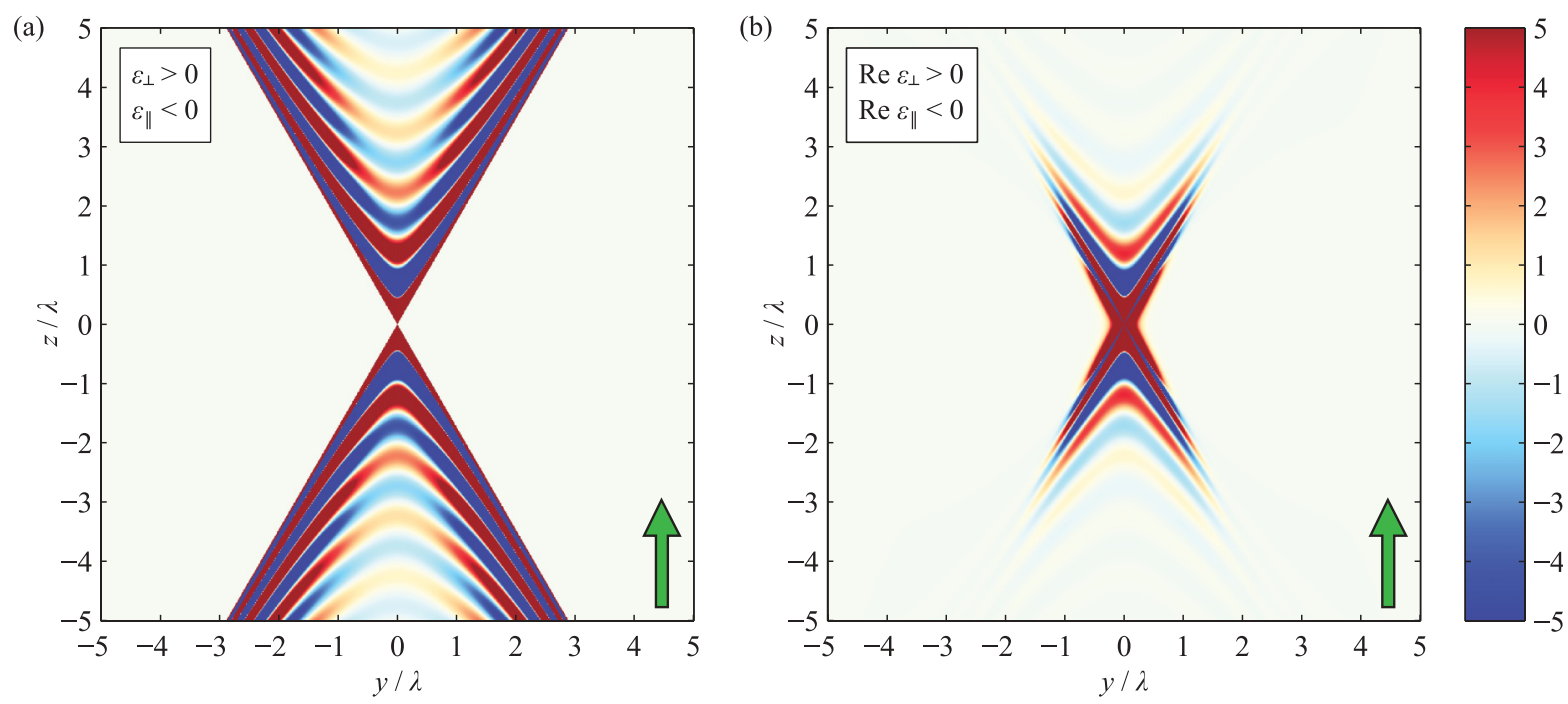

FIG. 2. (Color online) Dipole field $\lambda^{3} E_{z}(0, y, z)$ in the hyperbolic medium with $\varepsilon_{\perp}=1, \varepsilon_{\|}=-3$ (a) and with $\varepsilon_{\perp}=1+0.2 i$, $\varepsilon_{\|}=-3+0.2 i$ (b). Dipole moment is parallel to the axis of anisotropy $\hat{\mathbf{z}}$. 
(a)

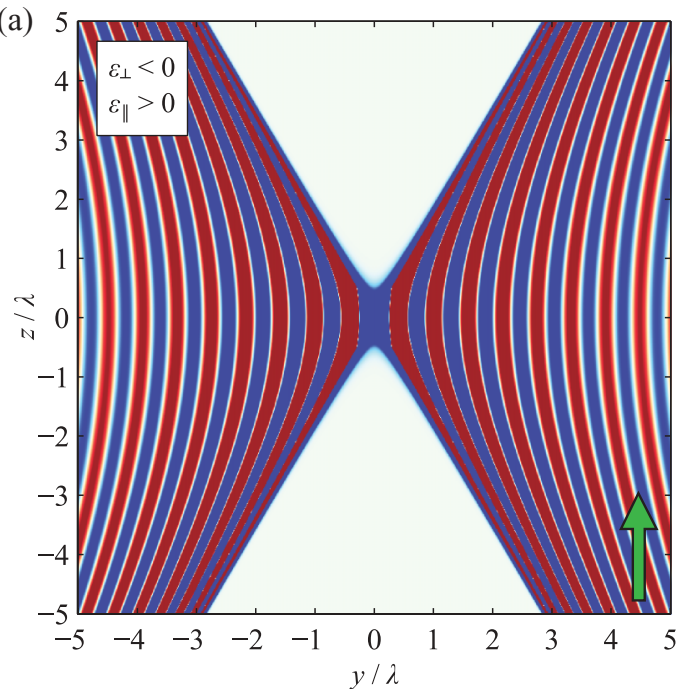

(b) 5

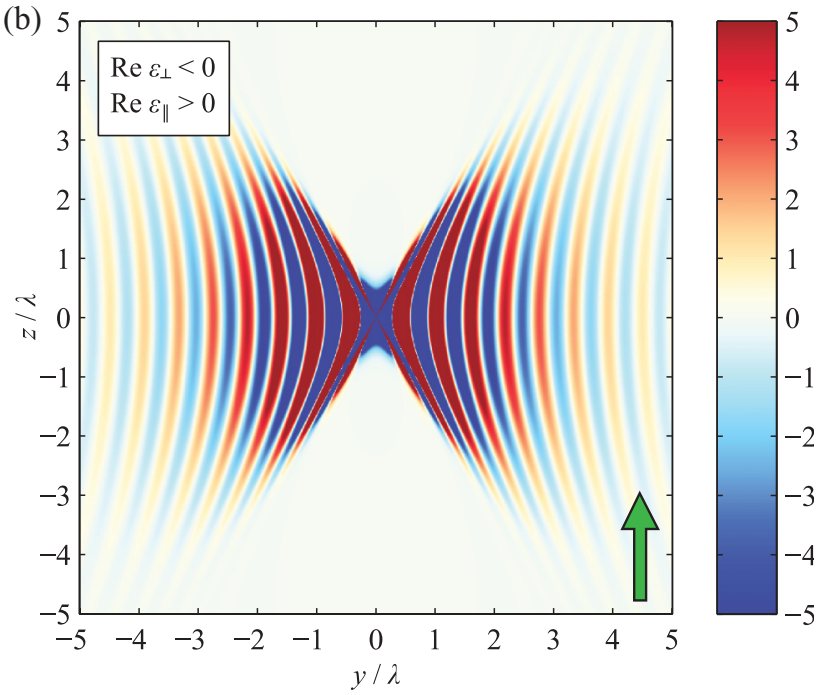

FIG. 3. (Color online) Dipole field $\lambda^{3} E_{z}(0, y, z)$ in the hyperbolic medium with $\varepsilon_{\perp}=-1, \quad \varepsilon_{\|}=3$ (a) and with $\varepsilon_{\perp}=-1+$ $0.2 i, \varepsilon_{\|}=3+0.2 i$ (b). Dipole moment is parallel to the axis of anisotropy $\hat{\mathbf{z}}$.

we obtain from Eqs. (7) and (21) the following result for the regular part of the electric field:

$$
\begin{aligned}
{[\mathbf{E}(\mathbf{r})]_{\mathrm{reg}}=} & p k^{2} \sqrt{\varepsilon_{\perp}} \frac{e^{i k r_{e}}}{r_{e}}\left(1-\frac{1}{i k r_{e}}-\frac{1}{k^{2} r_{e}^{2}}\right) \hat{\mathbf{z}} \\
& -p k^{2} \sqrt{\varepsilon_{\perp}} \frac{e^{i k r_{e}}}{r_{e}^{3}}\left(1-\frac{3}{i k r_{e}}-\frac{3}{k^{2} r_{e}^{2}}\right)(\mathbf{r} \cdot \hat{\mathbf{z}})(\hat{\epsilon} \mathbf{r}) .
\end{aligned}
$$

The Cartesian representation of this expression is presented in Appendix A. We see that the axial dipole emits only TMpolarized (extraordinary) waves. The calculated cross section of the electric field in the $y z$ plane is presented in Figs. 2 and 3 for both cases of the hyperbolic medium illustrated in Fig. 1: $\operatorname{Re} \varepsilon_{\|}<0, \operatorname{Re} \varepsilon_{\perp}>0$ and $\operatorname{Re} \varepsilon_{\|}>0, \operatorname{Re} \varepsilon_{\perp}<0$, respectively.
The electric-field pattern has a distinct conelike shape: the waves are emitted only within the polar angles $\theta$, satisfying $\varepsilon_{\|} \sin ^{2} \theta+\varepsilon_{\perp} \cos ^{2} \theta>0$. This radiation pattern, characteristic for the hyperbolic medium, survives even when the losses are introduced; see Figs. 2(b) and 3(b). An interesting feature of Eq. (28), revealed in Fig. 2, is that $\left|E_{z}(0,0, z)\right|$ is not zero for axial dipole orientation and decays as $1 /|z|$. This means that in the anisotropic medium the field is nonzero even along the direction of the dipole $\hat{\mathbf{z}}$.

\section{B. Dipole orthogonal to the axis of anisotropy}

Here we consider the case $\mathbf{p}=p \hat{\mathbf{y}}$. Taking into account that

$$
\hat{\epsilon} \cdot \hat{\mathbf{y}}=\varepsilon_{\|} \hat{\mathbf{y}}, \quad(\hat{\epsilon} \mathbf{r}) \cdot \hat{\mathbf{y}}=\varepsilon_{\|} \mathbf{r} \cdot \hat{\mathbf{y}},
$$

(a)

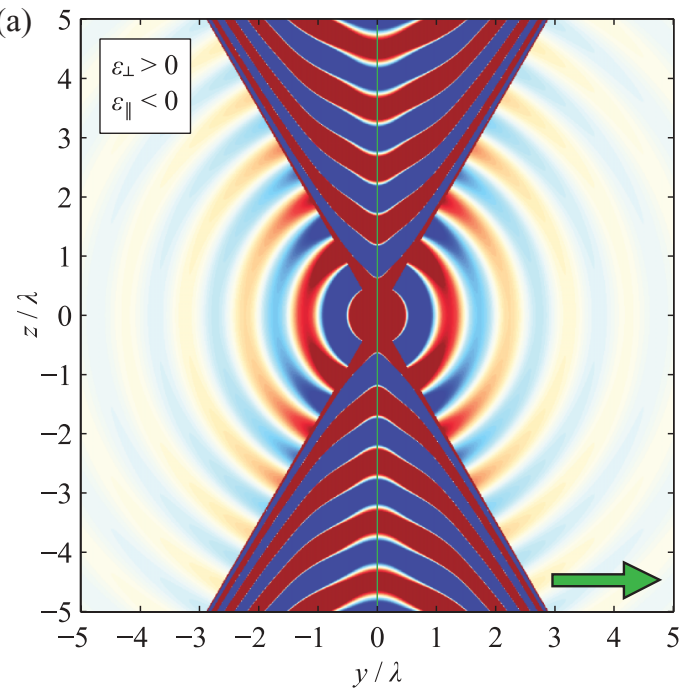

(b)

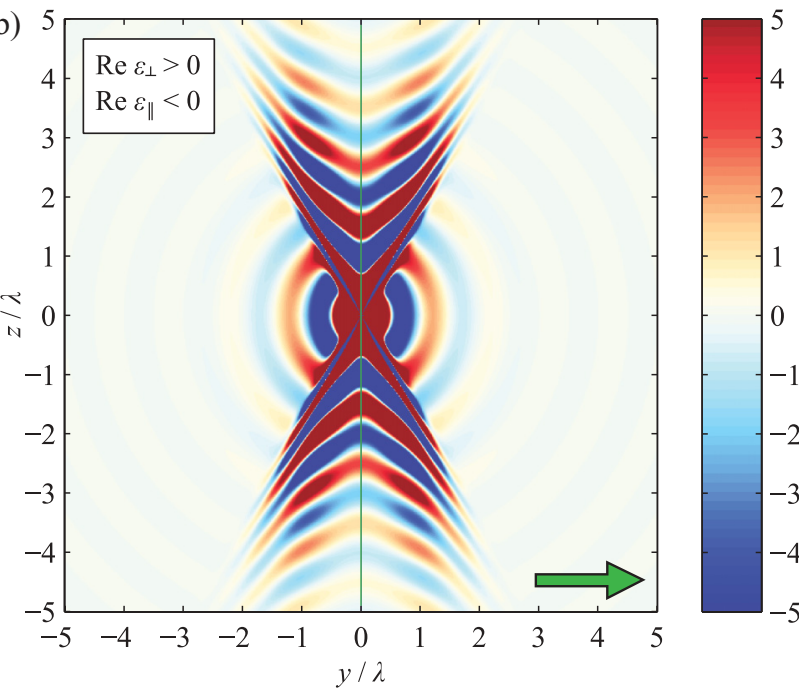

FIG. 4. (Color online) Dipole field $\lambda^{3} E_{y}(0, y, z)$ in the hyperbolic medium with $\varepsilon_{\perp}=1, \varepsilon_{\|}=-3$ (a) and with $\varepsilon_{\perp}=1+0.2 i, \varepsilon_{\|}=-3+0.2 i$ (b). Dipole moment is orthogonal to the axis of anisotropy $\hat{\mathbf{z}}$. 
(a)

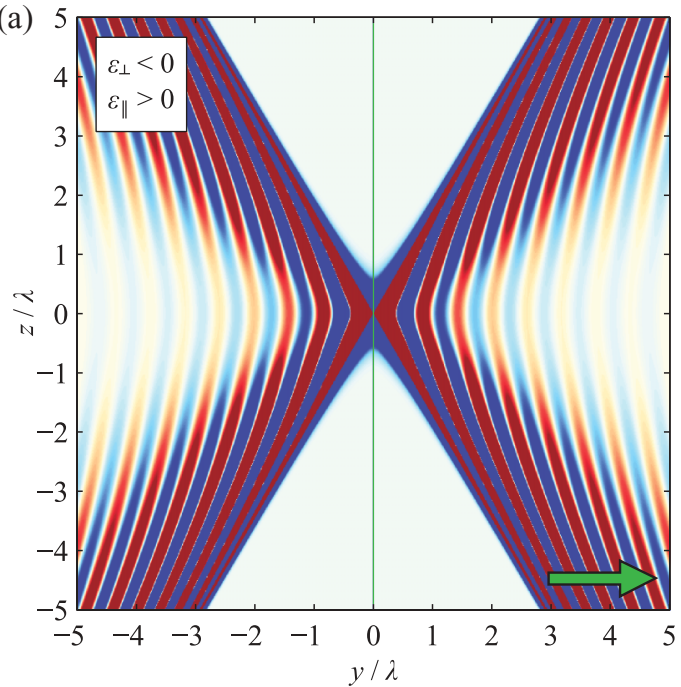

(b) 5

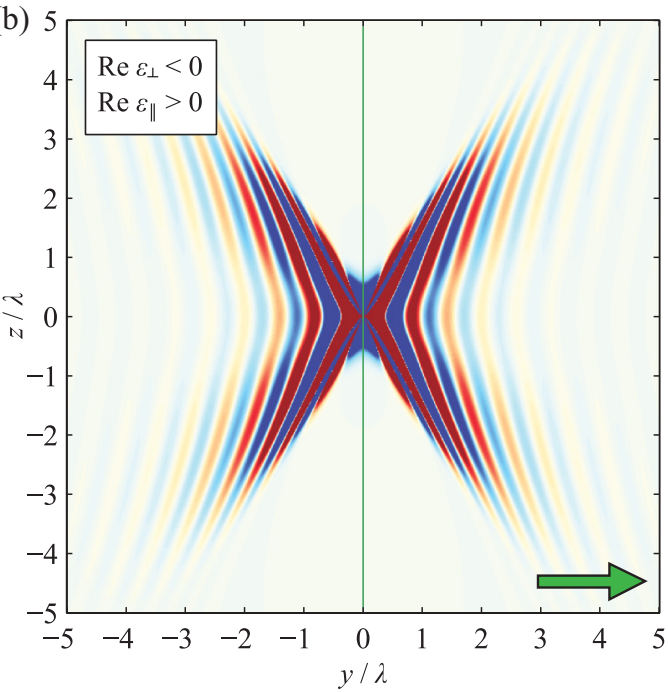

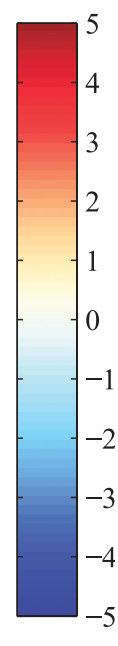

FIG. 5. (Color online) Dipole field $\lambda^{3} E_{y}(0, y, z)$ in the hyperbolic medium with $\varepsilon_{\perp}=-1, \varepsilon_{\|}=3$ (a) and with $\varepsilon_{\perp}=-1+0.2 i, \varepsilon_{\|}=3+0.2 i$ (b). Dipole moment is orthogonal to the axis of anisotropy $\hat{\mathbf{z}}$.

we obtain from (7), (21) the following result:

$$
\begin{aligned}
& {[\mathbf{E}(\mathbf{r})]_{\mathrm{reg}}} \\
& =p k^{2} \frac{\varepsilon_{\|}}{\sqrt{\varepsilon_{\perp}}} \frac{e^{i k r_{e}}}{r_{e}}\left(1-\frac{1}{i k r_{e}}-\frac{1}{k^{2} r_{e}^{2}}\right) \hat{\mathbf{y}} \\
& \quad-p k^{2} \frac{\varepsilon_{\|}}{\sqrt{\varepsilon_{\perp}}} \frac{e^{i k r_{e}}}{r_{e}^{3}}\left(1-\frac{3}{i k r_{e}}-\frac{3}{k^{2} r_{e}^{2}}\right)(\mathbf{r} \cdot \hat{\mathbf{y}})(\hat{\epsilon} \mathbf{r}) \\
& \quad+p k^{2}\left(\sqrt{\varepsilon_{\perp}} \frac{e^{i k r_{o}}}{r_{o}}-\frac{\varepsilon_{\|}}{\sqrt{\varepsilon_{\perp}}} \frac{e^{i k r_{e}}}{r_{e}}\right) \frac{[(\mathbf{r} \times \hat{\mathbf{z}}) \cdot \hat{\mathbf{y}}](\mathbf{r} \times \hat{\mathbf{z}})}{(\mathbf{r} \times \hat{\mathbf{z}})^{2}} \\
& \quad-i p k \frac{1}{\sqrt{\varepsilon_{\perp}}} \frac{e^{i k r_{o}}-e^{i k r_{e}}}{(\mathbf{r} \times \hat{\mathbf{z}})^{2}}\left(\hat{\mathbf{y}}-\frac{2[(\mathbf{r} \times \hat{\mathbf{z}}) \cdot \hat{\mathbf{y}}](\mathbf{r} \times \hat{\mathbf{z}})}{(\mathbf{r} \times \hat{\mathbf{z}})^{2}}\right) .
\end{aligned}
$$

The Cartesian representation of the last expression is presented in Appendix B. For this geometry both TE and TM polarized waves are emitted. Equation (29) includes the denominator $(\mathbf{r} \times \hat{\mathbf{z}})^{2} \equiv x^{2}+y^{2}$ which turns to zero at the line $x=y=0$. However, careful analysis of Eq. (29) ensures that the electric field is continuous at this line since diverging TE and TM wave contributions cancel each other. The calculated emission pattern is presented in Figs. 4 and 5 for different signs of dielectric constants. The most interesting results are manifested for $\operatorname{Re} \varepsilon_{\|}<0, \operatorname{Re} \varepsilon_{\perp}>0$ (Fig. 4), when the electric field is a distinct superposition of the conic pattern due to the TM waves emission and elliptic pattern due to the TE waves. In the second case $\operatorname{Re} \varepsilon_{\|}>0, \operatorname{Re} \varepsilon_{\perp}<0$ (Fig. 5) the TE waves contribution leads just to the spatial modulation of the conic radiation pattern. Similar to the case of the axial dipole orientation, the far field is present even along the dipole direction.

Interesting results are also revealed in the Poynting vector distribution, shown in Figs. 6 and 7. The Poynting vector is found as $\mathbf{S}=c /(4 \pi) \operatorname{Re} \mathbf{E} \times \mathbf{H}^{*}$; explicit expressions for magnetic field $\mathbf{H}$ are presented in Appendixes A and B. The Poynting vector pattern inherits the conical shape from the
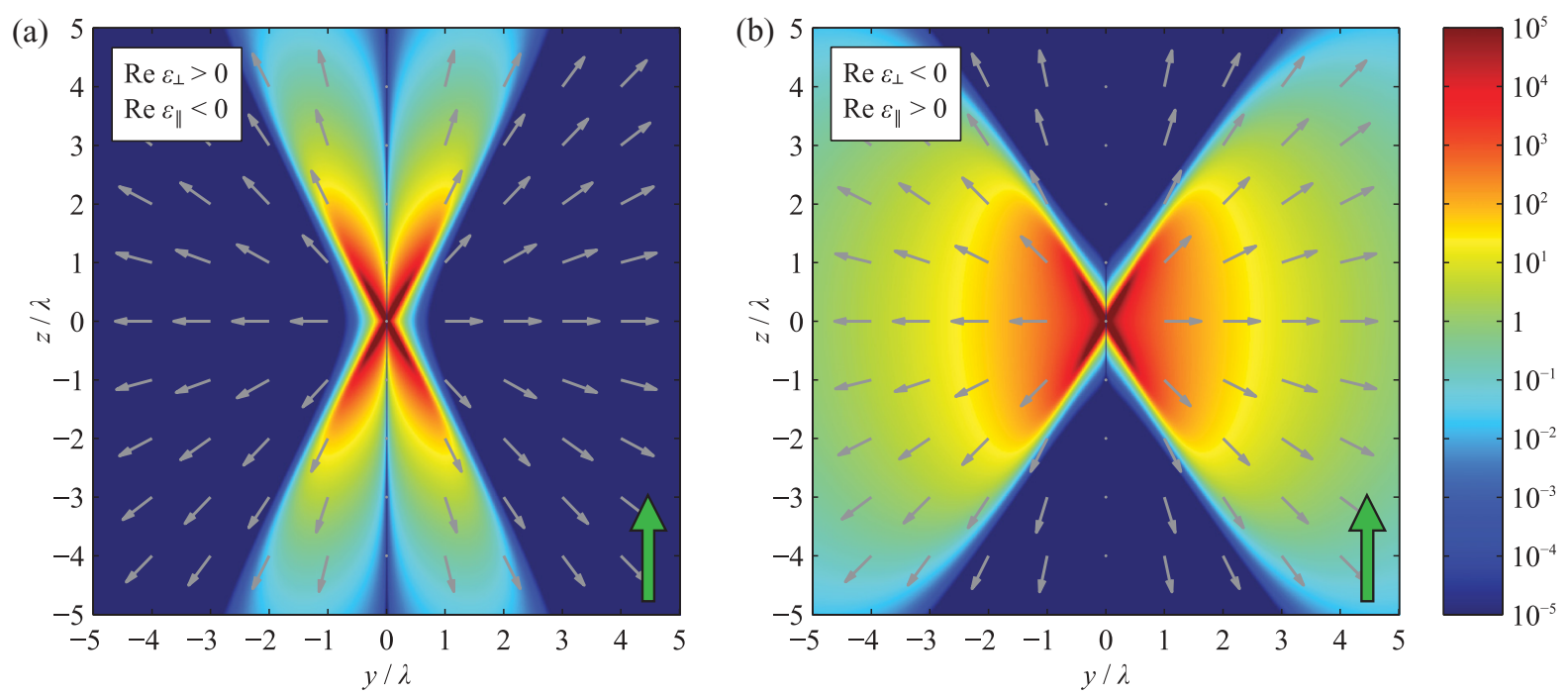

FIG. 6. (Color online) Poynting vector $4 \pi /\left(c \lambda^{6}\right) \mathbf{S}(0, y, z)$ in the hyperbolic medium with $\varepsilon_{\perp}=1+0.2 i$, $\varepsilon_{\|}=-3+0.2 i$ (a) and with $\varepsilon_{\perp}=-1+0.2 i, \varepsilon_{\|}=3+0.2 i$ (b). Dipole moment is parallel to the axis of anisotropy $\hat{\mathbf{z}}$. 

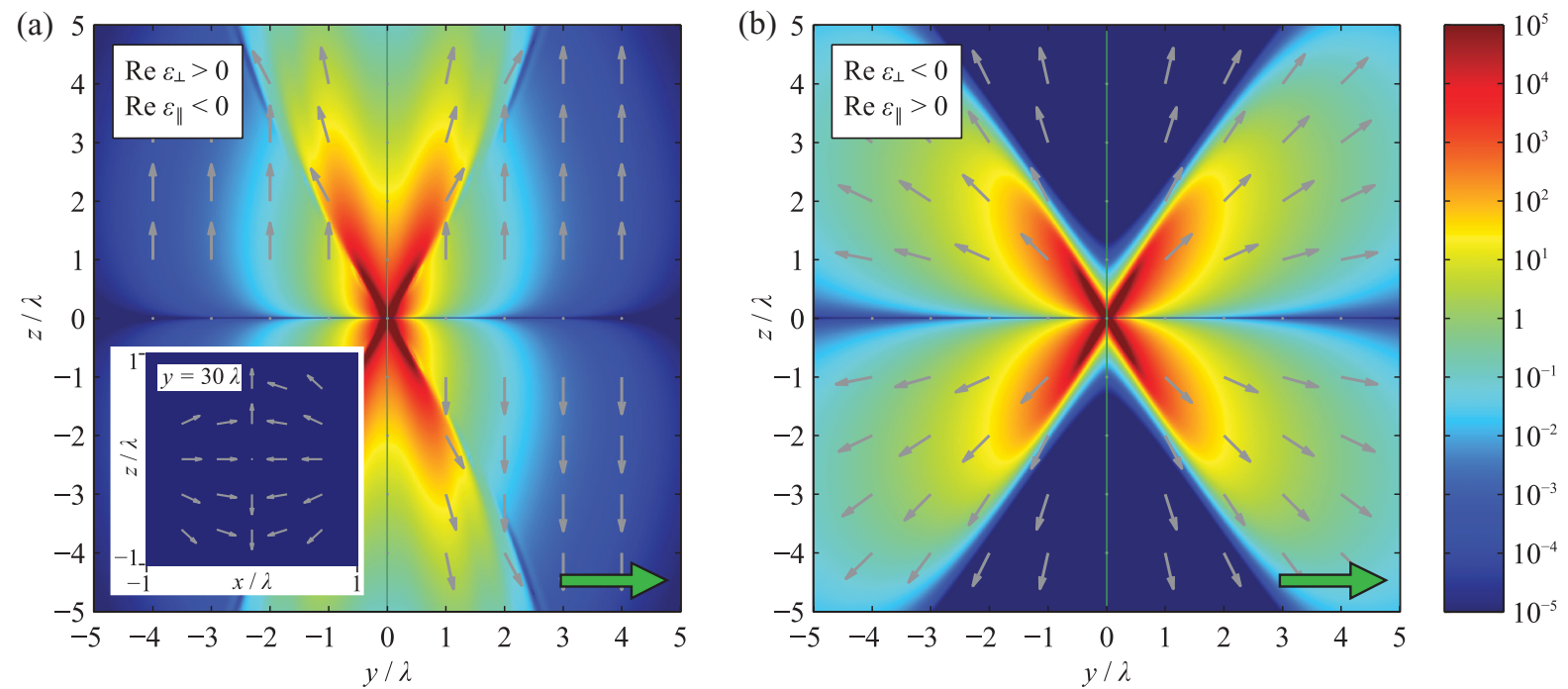

FIG. 7. (Color online) Poynting vector $4 \pi /\left(c \lambda^{6}\right) \mathbf{S}(0, y, z)$ in the hyperbolic medium with $\varepsilon_{\perp}=1+0.2 i$, $\varepsilon_{\|}=-3+0.2 i$ (a) and with $\varepsilon_{\perp}=-1+0.2 i, \varepsilon_{\|}=3+0.2 i$ (b). Dipole moment is orthogonal to the axis of anisotropy $\hat{\mathbf{z}}$. Inset in panel (a) illustrates the Poynting vector distribution in the $x z$ plane for $y=30 \lambda$.

electric field. Its largest values are achieved at the conical surface $r_{e}^{2}=0$. Interesting features are observed for the Poynting vector distribution in the case of perpendicular dipole orientation and $\operatorname{Re} \varepsilon_{\|}<0$, illustrated in Fig. 7(a). In the region outside of the cone, $r_{e}^{2}<0$, the Poynting vector in the $y z$ plane is directed almost along the $z$ direction. The resulting pattern looks as if there is a line source located at $y=x=0$, which violates the energy conservation condition. However, this first impression is wrong. Close inspection of the Poynting vector distribution reveals that the line $y=x=0$ is a saddle point of the Poynting vector: the energy enters the line along the $x$ direction and leaves along the $z$ one. This behavior is illustrated in the $x z$ cross section of the Poynting vector distribution, shown in the inset of Fig. 7(a).

One can also prove this result analytically. Neglecting the evanescent terms $\propto \exp \left(-k\left|r_{e}\right|\right)$ we find the following expression for the Poynting vector for vanishing losses:

$$
\begin{aligned}
\mathbf{S}= & \frac{c}{4 \pi r^{3 / 2}\left(x^{2}+y^{2}\right)^{2} \sqrt{\varepsilon_{\perp}}}\left\{\hat{\mathbf{x}}\left[k^{2} \varepsilon_{\perp} x^{3}\left(x^{2}+y^{2}\right)+x\left(x^{2}-y^{2}\right)\right]\right. \\
& +\hat{\mathbf{y}}\left[k^{2} \varepsilon_{\perp} x^{2} y\left(x^{2}+y^{2}\right)+2 x^{2} y\right] \\
& \left.+\hat{\mathbf{z}}\left[k^{2} \varepsilon_{\perp} x^{2} z\left(x^{2}+y^{2}\right)+z\left(r^{2}+y^{2}\right)\right]\right\} .
\end{aligned}
$$

Direct differentiation of Eq. (30) demonstrates that $\nabla \cdot \mathbf{S}=$ 0 , i.e., the energy conservation law is fulfilled. In the limit $|x|,|z| \ll y, 1 / k$ we find $\mathbf{S} \propto(z \hat{\mathbf{z}}-x \hat{\mathbf{x}}) /\left|y^{4}\right|$, which confirms the existence of the saddle point in the Poynting vector pattern.

\section{CONCLUSIONS}

We have presented a general theory of the dipole emission in homogeneous hyperbolic media. Using both the Fourier space approach and electromagnetic scaling, we have obtained a general expression for the electromagnetic Green function, and demonstrated that the emission pattern is highly anisotropic. For dipole orientation parallel to the symmetry axis, only TM-polarized waves are excited and the emission pattern has a conelike shape with propagating waves present only within the cone $\varepsilon_{\|} \sin ^{2} \theta+\varepsilon_{\perp} \cos ^{2} \theta>0$, where $\theta$ is the polar angle. In the case of the perpendicular orientation, the electric field is given by a sum of TE-polarized and TM-polarized contributions, so the waves can propagate also outside the cone. We have revealed a singular term in the Green function, and have demonstrated that it is of crucial importance for the calculation of the radiative rate of light source embedded in a hyperbolic medium. In the conventional case, the singular term, proportional to the $\delta$ function, is usually neglected, and does not contribute to the Purcell factor. However, in hyperbolic media this singular term is complex even for vanishing losses, and determines the diverging radiative decay rate.

\section{ACKNOWLEDGMENTS}

This work has been supported by the Ministry of Education and Science of Russian Federation, the Dynasty Foundation, Russian Foundation for Basic Research, European project POLAPHEN, EPSRC (UK), and the Australian Research Council. The authors acknowledge useful discussions with S. I. Maslovski, I. Yu. Popov, and C. R. Simovski.

\section{APPENDIX A: CARTESIAN REPRESENTATION OF THE FIELD OF DIPOLE PARALLEL TO THE ANISOTROPY AXIS}

Cartesian components of the electric field Eq. (28) of the dipole oriented along the anisotropy axis $z$ read

$$
\begin{aligned}
E_{x}= & -p k^{2} \varepsilon_{\|} \sqrt{\varepsilon_{\perp}} \frac{e^{i k r_{e}}}{r_{e}^{3}}\left(1-\frac{3}{i k r_{e}}-\frac{3}{k^{2} r_{e}^{2}}\right) x z, \\
E_{y}= & -p k^{2} \varepsilon_{\|} \sqrt{\varepsilon_{\perp}} \frac{e^{i k r_{e}}}{r_{e}^{3}}\left(1-\frac{3}{i k r_{e}}-\frac{3}{k^{2} r_{e}^{2}}\right) y z, \\
E_{z}= & p k^{2} \varepsilon_{\|} \sqrt{\varepsilon_{\perp}} \frac{e^{i k r_{e}}}{r_{e}^{3}} \\
& \times\left[x^{2}+y^{2}-\left(\frac{1}{i k r_{e}}+\frac{1}{k^{2} r_{e}^{2}}\right)\left(x^{2}+y^{2}-\frac{2 \varepsilon_{\perp}}{\varepsilon_{\|}} z^{2}\right)\right],
\end{aligned}
$$

where $r_{e}=\sqrt{\varepsilon_{\|}\left(x^{2}+y^{2}\right)+\varepsilon_{\perp} z^{2}}$. A similar result has been obtained in Ref. [33]. The magnetic field can be found as 


$$
\begin{aligned}
\mathbf{H}=-(i / k) \nabla & \times \mathbf{E}: \\
H_{x} & =p k^{2} \varepsilon_{\|} \sqrt{\varepsilon_{\perp}} \frac{e^{i k r_{e}}}{r_{e}^{2}}\left(1-\frac{1}{i k r_{e}}\right) y, \\
H_{y} & =-p k^{2} \varepsilon_{\|} \sqrt{\varepsilon_{\perp}} \frac{e^{i k r_{e}}}{r_{e}^{2}}\left(1-\frac{1}{i k r_{e}}\right) x, \\
H_{z} & =0 .
\end{aligned}
$$

\section{APPENDIX B: CARTESIAN PRESENTATION OF THE FIELD OF DIPOLE ORTHOGONAL TO THE ANISOTROPY AXIS}

Cartesian components of Eq. (29) for the dipole oriented along $y$ axis, perpendicular to the anisotropy axis $z$, read [33]

$$
\begin{gathered}
E_{x}=-p k^{2} \frac{\varepsilon_{\|}^{2}}{\sqrt{\varepsilon_{\perp}}} \frac{e^{i k r_{e}}}{r_{e}^{3}}\left(1-\frac{3}{i k r_{e}}-\frac{3}{k^{2} r_{e}^{2}}\right) x y-p k^{2}\left(\sqrt{\varepsilon_{\perp}} \frac{e^{i k r_{o}}}{r_{o}}-\frac{\varepsilon_{\|}}{\sqrt{\varepsilon_{\perp}}} \frac{e^{i k r_{e}}}{r_{e}}\right) \frac{x y}{x^{2}+y^{2}}-i p k \frac{1}{\sqrt{\varepsilon_{\perp}}}\left(e^{i k r_{o}}-e^{i k r_{e}}\right) \frac{2 x y}{\left(x^{2}+y^{2}\right)^{2}}, \\
E_{y}=p k^{2} \frac{\varepsilon_{\|}^{2}}{\sqrt{\varepsilon_{\perp}}} \frac{e^{i k r_{e}}}{r_{e}^{3}}\left[x^{2}+\frac{\varepsilon_{\perp}}{\varepsilon_{\|}} z^{2}-\left(\frac{1}{i k r_{e}}+\frac{1}{k^{2} r_{e}^{2}}\right)\left(x^{2}+\frac{\varepsilon_{\perp}}{\varepsilon_{\|}} z^{2}-2 y^{2}\right)\right]+p k^{2}\left(\sqrt{\varepsilon_{\perp}} \frac{e^{i k r_{o}}}{r_{o}}-\frac{\varepsilon_{\|}}{\sqrt{\varepsilon_{\perp}}} \frac{e^{i k r_{e}}}{r_{e}}\right) \frac{x^{2}}{x^{2}+y^{2}} \\
+i p k \frac{1}{\sqrt{\varepsilon_{\perp}}}\left(e^{i k r_{o}}-e^{i k r_{e}}\right) \frac{x^{2}-y^{2}}{\left(x^{2}+y^{2}\right)^{2}}, \quad E_{z}=-p k^{2} \varepsilon_{\|} \sqrt{\varepsilon_{\perp}} \frac{e^{i k r_{e}}}{r_{e}^{3}}\left(1-\frac{3}{i k r_{e}}-\frac{3}{k^{2} r_{e}^{2}}\right) y z,
\end{gathered}
$$

where

$$
r_{e}=\sqrt{\varepsilon_{\|}\left(x^{2}+y^{2}\right)+\varepsilon_{\perp} z^{2}}, \quad r_{o}=\sqrt{\varepsilon_{\perp}\left(x^{2}+y^{2}+z^{2}\right)} .
$$

The magnetic field reads

$$
\begin{aligned}
& H_{x}=-p k^{2} \varepsilon_{\|} \sqrt{\varepsilon_{\perp}} \frac{e^{i k r_{e}}}{r_{e}^{2}}\left(1-\frac{1}{i k r_{e}}\right) \frac{y^{2} z}{x^{2}+y^{2}}-p k^{2} \varepsilon_{\perp} \sqrt{\varepsilon_{\perp}} \frac{e^{i k r_{o}}}{r_{o}^{2}}\left(1-\frac{1}{i k r_{o}}\right) \frac{x^{2} z}{x^{2}+y^{2}}-i p k \sqrt{\varepsilon_{\perp}}\left(\frac{e^{i k r_{o}}}{r_{o}}-\frac{e^{i k r_{e}}}{r_{e}}\right) \frac{\left(x^{2}-y^{2}\right) z}{\left(x^{2}+y^{2}\right)^{2}}, \\
& H_{y}=p k^{2} \varepsilon_{\|} \sqrt{\varepsilon_{\perp}} \frac{e^{i k r_{e}}}{r_{e}^{2}}\left(1-\frac{1}{i k r_{e}}\right) \frac{x y z}{x^{2}+y^{2}}-p k^{2} \varepsilon_{\perp} \sqrt{\varepsilon_{\perp}} \frac{e^{i k r_{o}}}{r_{o}}\left(1-\frac{1}{i k r_{o}}\right) \frac{x y z}{x^{2}+y^{2}}-i p k \sqrt{\varepsilon_{\perp}}\left(\frac{e^{i k r_{o}}}{r_{o}}-\frac{e^{i k r_{e}}}{r_{e}}\right) \frac{2 x y z}{\left(x^{2}+y^{2}\right)^{2}}, \\
& H_{z}=p k^{2} \varepsilon_{\perp} \sqrt{\varepsilon_{\perp}} \frac{e^{i k r_{o}}}{r_{o}^{2}}\left(1-\frac{1}{i k r_{o}}\right) x .
\end{aligned}
$$

[1] L. B. Felsen and N. Marcuvitz, Radiation and Scattering of Waves (Prentice-Hall, Englewood Cliffs, NJ, 1972).

[2] D. R. Smith and D. Schurig, Phys. Rev. Lett. 90, 077405 (2003).

[3] D. R. Smith, P. Kolinko, and D. Schurig, J. Opt. Soc. Am. B 21, 1032 (2004).

[4] W. Cai, U. K. Chettiar, A. V. Kildishev, and V. M. Shalaev, Opt. Express 16, 5444 (2008).

[5] H. N. S. Krishnamoorthy, Z. Jacob, E. Narimanov, I. Kretzschmar, and V. M. Menon, Science 336, 205 (2012).

[6] Z. Jacob and V. M. Shalaev, Science 334, 463 (2011).

[7] E. M. Purcell, Phys. Rev. 69, 681 (1946).

[8] A. N. Poddubny, P. A. Belov, and Y. S. Kivshar, Phys. Rev. A 84, 023807 (2011).

[9] O. Kidwai, S. V. Zhukovsky, and J. E. Sipe, Opt. Lett. 36, 2530 (2011).

[10] I. Iorsh, A. Poddubny, A. Orlov, P. Belov, and Y. S. Kivshar, Phys. Lett. A 376, 185 (2012).

[11] W. Yan, M. Wubs, and N. Asger Mortensen, arXiv:1204.5413.

[12] A. N. Poddubny, P. A. Belov, P. Ginzburg, A. V. Zayats, and Y. S. Kivshar, Phys. Rev. B 86, 035148 (2012).

[13] J. Kim, V. P. Drachev, Z. Jacob, G. V. Naik, A. Boltasseva, E. E. Narimanov, and V. M. Shalaev, Opt. Express 20, 8100 (2012).

[14] R. K. Fisher and R. W. Gould, Phys. Rev. Lett. 22, 1093 (1969).
[15] S. Zhang, Y. Xiong, G. Bartal, X. Yin, and X. Zhang, Phys. Rev. Lett. 106, 243901 (2011).

[16] J. Yao, Z. Liu, Y. Liu, Y. Wang, C. Sun, G. Bartal, A. M. Stacy, and X. Zhang, Science 321, 930 (2008).

[17] M. A. Noginov, Y. A. Barnakov, G. Zhu, T. Tumkur, H. Li, and E. E. Narimanov, Appl. Phys. Lett. 94, 151105 (2009).

[18] L. V. Alekseyev, E. E. Narimanov, T. Tumkur, H. Li, Y. A. Barnakov, and M. A. Noginov, Appl. Phys. Lett. 97, 131107 (2010).

[19] C. L. Cortes, W. Newman, S. Molesky, and Z. Jacob, J. Opt. 14, 063001 (2012).

[20] A. A. Orlov, P. M. Voroshilov, P. A. Belov, and Y. S. Kivshar, Phys. Rev. B 84, 045424 (2011).

[21] A. V. Chebykin, A. A. Orlov, A. V. Vozianova, S. I. Maslovski, Y. S. Kivshar, and P. A. Belov, Phys. Rev. B 84, 115438 (2011).

[22] G. A. Wurtz, W. Dickson, D. O'Connor, R. Atkinson, W. Hendren, P. Evans, R. Pollard, and A. V. Zayats, Opt. Express 16, 7460 (2008).

[23] C. R. Simovski, P. A. Belov, A. V. Atrashchenko, and Y. S. Kivshar, Adv. Mater. 24, 4229 (2012).

[24] J. Sun, J. Zhou, B. Li, and F. Kang, Appl. Phys. Lett. 98, 101901 (2011).

[25] W. Vogel and D.-G. Welsch, Quantum Optics (Wiley, Weinheim, 2006).

[26] P. C. Clemmow, Proc. Inst. Elect. Eng. 110, 107 (1963). 
[27] H. C. Chen, Theory of Electromagnetic Waves: A Coordinatefree Approach, McGraw-Hill series in electrical engineering (McGraw-Hill, New York, 1983).

[28] W. Weiglhofer, Am. J. Phys. 56, 1095 (1988).

[29] W. Weiglhofer, Am. J. Phys. 57, 455 (1989).

[30] W. Weiglhofer, IEE Proc. H 137, 5 (1990).

[31] I. V. Lindell, Methods for Electromagnetic Field Analysis (Clarendon, Oxford University Press, Oxford, New York, 1992).

[32] P. Cottis, C. Vazouras, and C. Spyrou, IEEE Trans. Antennas Propag. 47, 195 (1999).

[33] A. Savchenko and O. Savchenko, Tech. Phys. 50, 1366 (2005).

[34] S. Sautbekov, I. Kanymgazieva, and P. Frangos, J. Appl. Electromagn. 10, 43 (2008).
[35] I. M. Gelfand and G. E. Shilov, Generalized Functions. Volume I: Properties and Operations (Academic, New York, 1964).

[36] L. Novotny and B. Hecht, Principles of Nano-Optics (Cambridge University Press, New York, 2006).

[37] E. L. Ivchenko, Optical Spectroscopy of Semiconductor Nanostructures (Alpha Science International, Harrow, UK, 2005).

[38] A. Yaghjian, Proc. IEEE 68, 248 (1980).

[39] C. Tai and A. Yaghjian, Proc. IEEE 69, 282 (1981).

[40] J. Wang, IEEE Trans. Antennas Propag. 30, 463 (1982).

[41] J. Franklin, Am. J. Phys. 78, 1225 (2010).

[42] C. P. Frahm, Am. J. Phys. 51, 826 (1983).

[43] P. C. Clemmow, Proc. Inst. Elect. Eng. 110, 101 (1963).

[44] I. Lindell, IEEE Trans. Antennas Propag. 36, 1382 (1988).

[45] I. Lindell, IEEE Trans. Antennas Propag. 38, 353 (1990). 